\title{
SPEED OF LIGHT IN NON-TRIVIAL VACUA
}

\author{
José I. Latorre*, Pedro Pascual and Rolf Tarrach** \\ Departament d'Estructura i Constituents de la Matèria \\ Facultat de Física, Universitat de Barcelona \\ Diagonal 647, 08028-Barcelona SPAIN \\ and IFAE
}

\begin{abstract}
We unify all existing results on the change of the speed of low-energy photons due to modifications of the vacuum, finding that it is given by a universal constant times the quotient of the difference of energy densities between the usual and modified vacua over the mass of the electron to the fourth power. Whether photons move faster or slower than $c$ depends only on the lower or higher energy density of the modified vacuum, respectively. Physically, a higher energy density is characterized by the presence of additional particles (real or virtual) in the vacuum whereas a lower one stems from the absence of some virtual modes. We then carry out a systematic study of the speed of propagation of massless particles for several field theories up to two loops on a thermal vacuum. Only low-energy massless particles corresponding to a massive theory show genuine modifications of their speed while remaining massless. All other modifications are mass-related, or running massrelated. We also develop a formalism for the Casimir vacuum which parallels the thermal one and check that photons travel faster than $c$ between plates.
\end{abstract}

\section{UB-ECM-PF 94}

* bitnet : latorre@ebubecm1

** bitnet : rolf@ebubecm1 


\section{Review of existing results}

The usual vacuum in a Quantum Field Theory is a state characterized by the absence of real particles and classical fields and by its Minkowskian geometry. Electromagnetic and gravitational fields as well as massless particles propagate though it with the same constant, Lorentz invariant speed, $c$. When the vacuum is modified, so is the speed of propagation of particles and fields. This quantum field theoretical effect has been analyzed within QED for low-energy photons in several cases that we now review.

Adler first studied the propagation of photons in an anisotropic vacuum given by an external constant uniform magnetic field, $\vec{B}$, and obtained the result $\left(\hbar=c=\epsilon_{0}=1\right)$ [1]

$$
\begin{array}{ll}
v_{\|}=1-\frac{8}{45} \alpha^{2} \frac{\vec{B}^{2}}{m_{e}^{4}} \sin ^{2} \theta & <1, \\
v_{\perp}=1-\frac{14}{45} \alpha^{2} \frac{\vec{B}^{2}}{m_{e}^{4}} \sin ^{2} \theta & <1,
\end{array}
$$

for polarizations respectively coplanar with and perpendicular to the plane defined by $\vec{B}$ and the direction of propagation. Here, $m_{e}$ is the mass of the electron, $\alpha$ is the fine structure constant and $\theta$ is the angle between $\vec{B}$ and the direction of propagation.

For a (homogeneous and isotropic) Robertson-Walker gravitational background with Friedmann cosmology, Drummond and Hathrell obtained [2]

$$
v=1+\frac{11}{45} \alpha G_{N} \frac{\rho+p}{m_{e}^{2}} \quad>1,
$$

where $G_{N}$ is Newton's constant and $\rho$ and $p$ are the energy density and pressure (which satisfy by the energy condition [3], $\rho+p>0$ ). Notice that the speed of light is now greater than $c$.

For a homogeneous and isotropic thermal vacuum, one of us first computed the change in the speed of light [4]. That result was corrected in Ref. [5] and is reobtained here in Sect. 5 with the result

$$
v=1-\frac{44 \pi^{2}}{2025} \alpha^{2} \frac{T^{4}}{m_{e}^{4}} \quad<1,
$$

where $T$ is the temperature and $k_{B}=1$.

Finally, Scharnhorst [6] and, independently, Barton [5] worked out the propagation of light in a (anisotropic and non-translational invariant) Casimir vacuum with the result

$$
v=1+\frac{11 \pi^{2}}{8100} \alpha^{2} \frac{1}{a^{4} m_{e}^{4}} \cos ^{2} \theta \quad>1,
$$

where $a$ is the distance between plates and $\theta$ is the angle between the direction of propagation and the normal to the plates. Again, photons propagate faster than $c$ in this case. 
There are several features worth mentioning in these four expressions. First, we note that the electron mass plays the role of an infrared regulator, so that the result is analytic for low energy and momenta. Also, $m_{e}$ provides the natural dimensions to compensate for those of the modified vacuum and, thus, sets the scale for this effect to be physically observable. All the above results are perturbative and should only be believed when small. The problem is that they are extremely small since any easily accessible values of $\vec{B}$, $G_{N}(\rho+p), T$ or $a^{-1}$ are negligible when compared to $m_{e}$. In other words, electrons are too heavy in this context.

A second relevant observation is that the modification of the polarization-averaged speed of light is always proportional to the numerical factor 11. This was already pointed out with respect to Eqs. (1.3), (1.4) in Ref. [5], where the author noted that this factor is common to all coherent light-by-light scattering [7]. Notice, however, that the same factor appears in Eq. (1.2) which is unrelated to light-by-light scattering, hinting at some deeper interpretation of the above results.

It is, nevertheless, hardly arguable that the most enticing feature of the above results is the sign of the modification of the speed of light: some vacua lead to $v<c$, some to $v>c$. This last possibility has motivated further study [5][8][9] [10]. The reader will find there a controversial discussion of phase, group and causal signals and, in particular, of the effects of $v>c$ on causality. Let us just say that, in accordance with ref. [2], we find no grounds for violation of microcausality.

The purpose of our work is to deepen and complete our understanding of the modifications of the speed of light. We do this by proceeding from the more physical to the more field-theoretical aspects of the problem. We first study the already known results presented above. We will give a unified and very physical interpretation of them which explains in an extremely simple manner the sign of the variation of the speed of light. We then concentrate on the thermal vacuum and consider the modification of the speed of (high and low energy) massless particles for different theories up to two loops. This will require a precise definition of what is meant by speed of massless particles which is presented in Sect. 3. A comprehensive one-loop study shows that field theories fall into two categories for the purpose of this study: those with an explicit mass, $m$, and those with an intrinsic mass scale, $\Lambda$. This is done in Sect. 4. In Sect. 5, the two-loop study for a theory of the first category, QED, is performed. This reproduces Eq. (1.3) at low energies and leads to a new result at high energies. The two-loop study for a theory of the second category, massless $g \phi^{4}$, is performed in Sect. 6. This is mainly of field theoretical interest as in the Standard Model only the quarkless QCD sector has no explicit mass. Confinement only allows massless particles beyond the deconfinement temperature which does not correspond to the low temperature cold heat bath situation we are considering here $(T \ll m$ or $T \ll \Lambda)$. Still, it is not excluded that these results will be of some relevance to photon propagation if almost massless pseudogoldstone bosons, like invisible axions, exist. In Sect. 7, a comparison of the thermal and Casimir vacua is performed. This allows us, first, to better understand the meaning of a modified vacuum and, second, 
to introduce a unified formalism which explains, both mathematically and theoretically, the different signs of the modifications of the speed of light. We finally summarize our most relevant results.

\section{The unified formula}

We mentioned in the previous section that all the polarization-averaged modifications of the speed of light are proportional to the numerical factor 11 and inversely proportional to $m_{e}^{4}$. This hints at the possibility of writing them in a unified form, which thus digs out the common phenomenon underlying the physics behind them. This is indeed so, as we now show.

Consider first the magnetic field vacuum. From Eq. (1.1) one finds the averaged speed of light

$$
v=\frac{1}{4} \int_{0}^{\pi} d \theta \sin \theta\left(v_{\|}(\theta)+v_{\perp}(\theta)\right)=1-\frac{22}{135} \alpha^{2} \frac{\vec{B}^{2}}{m_{e}^{4}},
$$

which, recalling that the energy density for a magnetic field is given by

$$
\rho_{B}=\frac{\vec{B}^{2}}{2}>0
$$

can be written as

$$
v=1-\frac{44}{135} \alpha^{2} \frac{\rho_{B}}{m_{e}^{4}} .
$$

For the Friedmann-Robertson-Walker vacuum, we recall that a radiation dominated universe, as corresponds to $T \ll m_{e}$, is described by $p=\frac{\rho}{3}$. Moreover, the gravitational energy density is negative

$$
\rho_{G}=-\rho<0
$$

so that Eq. (1.2) can be written as

$$
v=1-\frac{44}{135} \alpha\left(G_{N} m_{e}^{2}\right) \frac{\rho_{G}}{m_{e}^{4}}
$$

On its own, the cold temperature vacuum is characterized by its Planck energy density

$$
\rho_{T}=\frac{\pi^{2}}{15} T^{4}>0
$$

so that Eq. (1.3) reads

$$
v=1-\frac{44}{135} \alpha^{2} \frac{\rho_{T}}{m_{e}^{4}} .
$$


Finally, the averaged speed of light for the Casimir vacuum is, from Eq. (1.4),

$$
v=\frac{1}{2} \int_{0}^{\pi} d \theta \sin \theta v(\theta)=1+\frac{11 \pi^{2}}{24300} \alpha^{2}\left(\frac{1}{a m_{e}}\right)^{4} .
$$

Recalling the energy density of the Casimir vacuum [11],

$$
\rho_{a}=-\frac{\pi^{2}}{720 a^{4}}<0,
$$

the speed of light can be written as

$$
v=1-\frac{44}{135} \alpha^{2} \frac{\rho_{a}}{m_{e}^{4}} .
$$

Note that Eqs. (2.3), (2.7) and (2.10) are identical, and (2.5) also coincides with them if we substitute $G_{N} m_{e}^{2}$ by $\alpha$. We therefore find the following remarkable result: the low energy modification of the speed of light is proportional to the ratio of the energy density of the modified vacuum (as compared to the usual one) over $m_{e}^{4}$, with a universal numerical coefficient and the corresponding coupling constants. If the modified vacuum has a higher energy density, then $v<c$. If it is lower, then $v>c$. This is what explains the sign of the change of the speed of light.

The general equation we have found for the speed of light in a modified vacuum allows us to make further predictions. As an instance, low-energy photons should travel in an external electric field, $\vec{E}$, with a speed

$$
v=1-\frac{22}{135} \alpha^{2} \frac{\vec{E}^{2}}{m_{e}^{4}} .
$$

Another remarkable consequence of the general equation we have found is the fact that only for an asymptotically flat universe would the speed of light remain $c$. Matter, radiation and gravitational energy add to zero, which is the relevant physical quantity that affects the velocity of low-energy photons at this order of perturbation theory.

Let us finish this section mentioning one surprising feature. Notice that the numerical coefficient is the same for the gravitational vacuum as for the others, in spite of its being unrelated to light-by-light scattering. Furthermore this factor 11 also appears in the coefficient of the Euler-Poincaré characteristic spin $\frac{1}{2}$ contribution to the gravitational trace anomaly [11][12]. These connections will be discussed elsewhere.

\section{Definitions and framework}

Let us first define what we mean by speed of light, or better, speed of a massless particle. Consider a massless scalar particle which moves in a thermal vacuum. Its propagator is given by

$$
\frac{Z^{-1}}{q_{0}^{2}-\overrightarrow{q^{2}}-\Pi\left(q_{0}^{2}-\overrightarrow{q^{2}}\right)-\Pi_{T}\left(q_{0}^{2}, \overrightarrow{q^{2}}\right)} \quad, \quad \Pi(0)=0,
$$


where $Z$ is the field renormalization constant, $\Pi$ the zero temperature self-energy and $\Pi_{T}$ its temperature correction. The separation of $q_{0}^{2}$ and $\overrightarrow{q^{2}}$ in $\Pi_{T}$ reflects the Lorentz-symmetry breaking induced by the heat bath.

We will define the speed of a massless particle for real, rather than virtual, particles. This is because for gauge theories definitions on the pole, even at finite temperature, ensure gauge invariance [13]. The energy-momentum dispersion relation which characterizes the propagation of a real particle is obtained from imposing that the denominator of Eq. (3.1) vanishes. This leads to the implicit solution $q_{0}=E(q), q \equiv|\vec{q}|$. At one-loop order one finds an explicit solution independent of $\Pi$,

$$
E^{2}(q)=q^{2}+\Pi_{T}^{(1)}\left(q^{2}, q^{2}\right)
$$

Note that the standard vacuum polarization, $\Pi$, does not break Lorentz invariance and, thus, does not change the energy-momentum dispersion relation.

In general, perturbation theory allows to obtain an explicit solution

$$
E^{2}(q)=q^{2}+f(q, T, m)
$$

where $f(q, T, m)$ depends on $\Pi$ and $\Pi_{T}$. Its dependence on momentum, temperature and virtual particle mass has been specified. If the theory is massless, $m=0$, an intrinsic mass scale, $\Lambda$, will appear beyond one loop through the running of the coupling constant. Then, $f(q, T, m)$ should be understood as $f(q, T, \Lambda)$. Certainly the dependencies on $m$ and $\Lambda$ are different.

The speed of the massless particle is then given (as for photons traveling through a medium that changes the relation between $E$ and $p$ ) by

$$
v(q) \equiv \frac{\partial E}{\partial q}=\frac{q+\frac{1}{2} f^{\prime}(q, T, m)}{\sqrt{q^{2}+f(q, T, m)}} \quad, \quad f^{\prime}(q, T, m) \equiv \frac{\partial}{\partial q} f(q, T, m)
$$

Expansions of potential relevance are

$$
v \simeq 1-\frac{1}{2 q^{2}}\left(f(q, T, m)-q f^{\prime}(q, T, m)\right) \quad, \quad q^{2} \gg f, q \gg f^{\prime}
$$

with $v \simeq 1$,

$$
v \simeq \frac{f^{\prime}(q, T, m)}{2 \sqrt{f(q, T, m)}} \quad, \quad f^{\prime} \gg q, f \gg q^{2}
$$

and

$$
v \simeq \frac{q}{\sqrt{f(q, T, m)}} \quad, \quad f^{\prime}(q, T, m) \ll q, f \gg q^{2}
$$

with $v \rightarrow 0$, in spite of going, to one loop, as the inverse of the square root of the coupling constant. 
Three particular cases will be of interest. First, let $f$ be constant in $q$,

$$
f(q, T, m) \equiv \lambda^{2}(T, m) .
$$

The whole effect of the heat bath is, then, to give a mass to the particle, which however remains free, see Eq. (2.3). The speed of the particle is modified as corresponds to acquiring a mass: from Eq. (3.5) and Eq. (3.7), as Eq. (3.6) never applies,

$$
\begin{array}{rlrl}
v & \simeq 1-\frac{\lambda^{2}(T, m)}{2 q^{2}} & & \\
v \simeq \frac{q}{\lambda(T, m)} & & q \gg \lambda \\
& , \quad \lambda \gg q
\end{array}
$$

Second, let us consider the case when no mass is induced at low energies,

$$
\lim _{q \rightarrow 0} f(q, T, m)=0 .
$$

Then, $v(q)$ is a genuine modification of the speed of the massless particle at low energies, since it remains massless. The dispersion relation is usually non-free. If, furthermore,

$$
f(q, T, m) \sim 0\left(q^{2}\right) \quad, \quad q \ll T
$$

(recall that always $T \ll m$ ), then Eq. (3.5) always holds in perturbation theory and

$$
v(q \ll T) \simeq 1+\frac{f^{\prime \prime}(0, T, m)}{4} \quad, \quad f^{\prime \prime} \ll 1 .
$$

This is the situation which corresponds to the results reviewed in the introduction. In the particular case $f(q, T, m) \sim q^{2}$ for all momenta the dispersion relation would be free but the speed of the massless particle would have changed according to Eq. (3.12) for all values of $q$, i.e. it would be a different constant now. We have not encountered this situation in any of the theories we have investigated.

Third, let us consider the case when no mass is induced at high energies,

$$
\lim _{q \rightarrow \infty} f(q, T, m)=0 .
$$

Then, at high energies Eq. (3.5) holds and represents a genuine modification of the speed of the massless particle at high energies, for it remains massless. The dispersion relation is never free.

We have been implicitly assuming that $f(q, T, m)$ is real. This is, however, usually not so [14], but imaginary contributions of the self energy are not relevant to the issue of the speed of the massless particle. Also, no zero momentum ambiguities [15][16] appear in what follows. 
For massless Dirac fermions the propagator is given by

$$
\frac{Z^{-1}}{q_{0} \gamma_{0}-\vec{q} \cdot \vec{\gamma}-\Sigma\left(q_{0}^{2}-q^{2}\right)\left(q_{0} \gamma_{0}-\vec{q} \cdot \vec{\gamma}\right)-\Sigma_{s, T}\left(q_{0}^{2}, q^{2}\right) q_{0} \gamma_{0}+\Sigma_{v, T}\left(q_{0}^{2}, q^{2}\right) \vec{q} \cdot \vec{\gamma}}
$$

with a notation similar to the used in Eq. (3.1). Notice that no temperature contribution of other Dirac matrix structures have been included, because they will not appear for the theories and perturbative order considered in this work. The dispersion relation is given by

$$
q_{0}^{2}\left(1-\Sigma\left(q_{0}^{2}-q^{2}\right)-\Sigma_{s, T}\left(q_{0}^{2}, q^{2}\right)\right)^{2}=q^{2}\left(1-\Sigma\left(q_{0}^{2}-q^{2}\right)-\Sigma_{v, T}\left(q_{0}^{2}, q^{2}\right)\right)^{2}
$$

At one-loop order this leads to

$$
E^{2}(q)=q^{2}\left(1+2 \Sigma_{s, T}^{(1)}\left(q^{2}, q^{2}\right)-2 \Sigma_{v, T}^{(1)}\left(q^{2}, q^{2}\right)\right)
$$

or, with the notation of Eq. (3.3), to

$$
f(q, T, m)=2 q^{2}\left(\Sigma_{v, T}^{(1)}\left(q^{2}, q^{2}\right)-\Sigma_{v, T}^{(1)}\left(q^{2}, q^{2}\right)\right) .
$$

For massless gauge bosons the corresponding analysis is slightly more complicated [4]. The result to one-loop order is

$$
E^{2}(q)=q^{2}-\frac{1}{2}\left(\delta^{i j}-\hat{q}^{i} \hat{q}^{j}\right) \Pi_{i j, T}^{(1)}\left(q^{2}, q^{2}\right)
$$

where $\hat{q}^{i}$ is the spatial unit vector and $\Pi_{i j, T}$ are the spatial components of the temperature contribution to the self-energy tensor $\Pi_{\mu v}$, so that

$$
f(q, T, m)=-\frac{1}{2}\left(\delta^{i j}-\hat{q}^{i} \hat{q}^{j}\right) \Pi_{i j, T}^{(1)}\left(q^{2}, q^{2}\right) .
$$

The one-loop expressions also hold at, say, two loops when there are no one-loop temperature corrections. This will happen for our two-loop QED study, because we will always neglect the thermal contribution due to massive virtual particles which are always exponentially suppressed by a huge Boltzmann factor. This is because we consider, as said in the introduction, only cold heat baths, $T \ll m$.

Summing up: our test particle is real and massless and we only thermalize massless virtual particles, so that if the theory has a massive particle it only enters in the form of a virtual non-thermalized contribution.

\section{One-loop results}

Let us consider the propagation of massless particles in a cold heat bath, using perturbation theory at one loop. We are here not concerned with hot heat baths, which require 
a different formalism [17], because our initial motivation was the smallness of the effects reviewed in the introduction, and their extension to high energies and massless theories, but without changing the conditions defining the vacuum. We will use the real time formalism [18], which at the one-loop level reduces to the original one-component formalism [19]. Thus, a massless scalar boson propagator reads

$$
D_{T}\left(k, k^{\prime}\right)=\delta^{(4)}\left(k-k^{\prime}\right)\left[\frac{i}{k^{2}+i \eta}+\frac{2 \pi}{e^{\frac{\left|k_{0}\right|}{T}}-1} \delta\left(k^{2}\right)\right], \quad k^{2}=k_{0}^{2}-\vec{k}^{2}
$$

while a gauge boson propagator in the Feynman gauge is given by

$$
D_{T}^{\mu v}\left(k, k^{\prime}\right)=-g^{\mu v} D_{T}\left(k, k^{\prime}\right)
$$

For a massless Dirac fermion one has

$$
S_{T}\left(k, k^{\prime}\right)=\delta^{(4)}\left(k-k^{\prime}\right) \not k\left[\frac{i}{k^{2}+i \eta}-\frac{2 \pi}{e^{\frac{\left|k_{0}\right|}{T}}+1} \delta\left(k^{2}\right)\right]
$$

with $\not k \equiv k_{\mu} \gamma^{\mu}$. The above expressions separate the zero temperature from the finite temperature contributions. A heat bath has, of course, other effects on the propagation of a particle (e.g. diffusion), but the ones which cannot be told from the virtual particle effects, because they conserve energy and momentum, are included in the modified vacuum and are given by Eq. $(4.1,2,3)$.

Consider first massless $\left(m_{e}=0\right)$ QED. For the photon one finds from Eq. (3.19) and (4.3) that $f(q, T)$ is constant, i.e. Eq. (3.8) applies and

$$
\lambda_{\gamma}^{2}(T)=2 \frac{e^{2} T^{2}}{12}
$$

where we have stressed that any of the two virtual electrons thermalizes, and $e$ is the electron charge. For the massless electron, from Eq. (3.17), (4.2) and (4.3), again $f(q, T)$ comes out constant

$$
\lambda_{e}^{2}(T)=\frac{e^{2} T^{2}}{6}+\frac{e^{2} T^{2}}{12},
$$

where we have separated the thermalized photon from the thermalized electron contributions. The constancy of $f(q, T)$ at one loop turns out to be a general one-loop result for theories without explicit masses. Indeed, in $S U\left(N_{c}\right)$ Yang-Mills theory without quarks one obtains (ghosts are thermalized as scalar bosons)

$$
\lambda_{g}^{2}(T)=N_{c} \frac{g_{s}^{2} T^{2}}{3},
$$

where $N c$ is the number of colors and $g_{s}$ the strong coupling constant. Also for massless $\frac{g}{4 !} \phi^{4}$, where the tadpole is the only diagram which appears at one loop, the result is constant

$$
\lambda_{\phi}^{2}(T)=\frac{g T^{2}}{24}
$$


Consider now the other type of theories we are interested in: those with a virtual non-thermalized massive particle. At one-loop order there are no thermal contributions in QED, as the test particle, being massless, has to be the photon and the virtual ones are all massive electrons. This will be important when QED is studied at two loops in the next section. The Weinberg-Salam model has, however, diagrams which contribute, specifically the one with neutrinos both as test and thermalized particles and the $Z^{0}$ as the virtual massive particle. The result is

$$
f\left(q, T, M_{z}\right)=\frac{g_{W}^{2}}{2}\left[\frac{T^{2}}{6}+\frac{M_{z}^{2} T}{4 \pi^{2} q} \int_{0}^{\infty} d x \frac{1}{e^{x}+1} \ln \left|\frac{M_{z}^{2}-4 q T x}{M_{z}^{2}+4 q T x}\right|\right]
$$

where $g_{W} \equiv \frac{e}{2 \sin \theta_{W} \cos \theta_{W}}, \theta_{W}$ is Weinberg's angle and $M_{z}$ is the $Z^{0}$ mass. This satisfies Eq. (3.10) but not Eq. (3.13). Thus, the neutrino remains massless at low energies and its low energy velocity is modified according to Eq. (3.12),

$$
v\left(q T \ll M_{z}^{2}\right)=1-g_{W}^{2} \frac{7 \pi^{2}}{45}\left(\frac{T}{M_{z}}\right)^{4}+\mathcal{O}\left(\frac{q^{2} T^{6}}{M_{z}^{8}}\right),
$$

which is always a small correction for cold heat baths, while at high energies it goes like

$$
v\left(q T \gg M_{z}^{2}\right)=1-g_{W}^{2} \frac{1}{24}\left(\frac{T}{q}\right)^{2}+\mathcal{O}\left(\frac{M_{z}^{4}}{q^{4}}\right), \quad T \leq q,
$$

where we have specified the condition for perturbation theory to hold. Notice that Eq. (4.10) is like Eq. (3.9a) so that the neutrino has indeed acquired a mass at high energies. This can also be seen from the fact that Eq. (4.10) allows the limit $M_{z} \rightarrow 0$ to be taken, without being modified. But, as analyzed previously in this section, for $M_{z}=0$ the whole temperature effect should give the neutrino a mass. Indeed, putting $M_{z}=0$ in Eq. (4.8) gives (recall that still only neutrinos are thermalized)

$$
\lambda_{\nu}^{2}(T)=g_{W}^{2} \frac{T^{2}}{12}
$$

so that Eq. (4.10) can be written as

$$
v\left(q T \gg M_{z}^{2}\right)=1-\frac{\lambda_{\nu}^{2}(T)}{2 q^{2}}, \quad \lambda_{\nu} \ll q,
$$

exactly as Eq. (3.9a). Thus, the neutrino does not acquire a mass at low energies, but it does so at high energies, and the mass it acquires is precisely the one it would have gotten in the massless theory. In other words

$$
\lim _{q \rightarrow \infty} f\left(q, T, M_{z}\right)=f(q, T, 0)=\lambda_{\nu}^{2}(T)
$$


The result Eq. (4.9) (with $v<1$ and its quartic $T$ dependence), the result Eq. (4.10) with $v<1$ and its quadratic $T$ dependence) and its interpretation Eq. (4.12) as a mass effect are of general validity at one loop. One can easily check them in other examples, e.g. in an effective theory of photons and neutral massive scalars or pseudoscalars.

At one loop only two scenarios exist. In a massless theory the test particle acquires a mass and nothing else. Its speed is modified accordingly. In a massive theory the test particle remains massless at low energies and its speed is slowed down as $\left(\frac{T}{m}\right)^{4}$ at low energies. This can be interpreted as due to a change in the vacuum energy density as done in section 2. At high energies, where the mass of the theory should be irrelevant, it acquires a mass; precisely the one it would have acquired in the massless theory. Its speed is slowed down accordingly.

From the results presented in this section, only the one corresponding to the WeinbergSalam theory can be straight-forwardly used for phenomenological estimations. The corrections to the speed of neutrinos are even smaller than the ones of photons reviewed in the introduction.

\section{Two-loop QED}

We now turn to the study of the modification of the speed of photons in QED at two loops. The calculation has already been performed in the literature for low-energy photons [4][6]. In such a case, the result can be obtained from the Euler-Heisenberg energy density, which encodes the fermion box diagram for constant external fields. At high energies, there is no possibility to circumvent the use of the full machinery of quantum field theory at finite temperature. Therefore, we proceed now to perform that first principles computation.

For QED, because in the limit in which only massless particles thermalize $\Pi_{i j, T}^{(1)}=0$, one can use at two loops all the one-loop formulae and techniques. Thus from Eq. (3.18) and Eq. (4.2) one obtains [4]

$$
f\left(q, T, m_{e}\right)=-i e^{4} \pi \int \frac{d^{4} k}{(2 \pi)^{4}} \frac{1}{e^{\frac{\left|k_{0}\right|}{T}}-1} \delta\left(k^{2}\right) A\left(q, k, m_{e}\right)
$$

where the integrand, which corresponds to the electron box loop, is given by

$$
\begin{aligned}
& \quad A\left(q, k, m_{e}\right)=\int \frac{d^{n} p}{(2 \pi)^{n}}\left[\frac{4(n-2)^{2}}{D(0) D(0)}-\frac{16(n-2)}{D(0) D(k)}-\frac{2(n-2)(n+4)}{D(0) D(k+q)}\right. \\
& +\frac{2(n-2)(n+8)}{D(0) D(q-k)}-\frac{64 q \cdot k}{D(0) D(q) D(k)}-\frac{32 q \cdot k}{D(0) D(k) D(q+k)}-\frac{64 m_{e}^{2}}{D(0) D(0) D(k)} \\
& \left.+\frac{64 m_{e}^{4}}{D(k) D(q) D(k+q)}+\frac{64 m_{e}^{4}}{D(0) D(0) D(k) D(q)}+\frac{16 m_{e}^{2}\left(5 q \cdot k+2 m_{e}^{2}\right)}{D(0) D(k) D(q) D(k+q)}\right]
\end{aligned}
$$

where $n$ is the dimension of space-time and

$$
D(k) \equiv(p-k)^{2}-m_{e}^{2}+i \eta
$$


The integral in Eq. (5.2) is ultraviolet (UV) convergent. Using Feynman parametrization, it is easy to carry out the fermionic momentum integral as well as all the parametric ones but the last. This last parametric integral would lead to complicate dilogarithmic expressions and, therefore, we prefer to give its result in terms of series,

$$
\begin{aligned}
A\left(q, k, m_{e}\right)=\frac{4 i}{\pi^{2}} \sum_{l=1}^{\infty} \frac{\Gamma^{2}(l)}{\Gamma(2 l+3)} & {\left[\left(12 l^{2}+43 l^{3}+50 l^{2}+26 l-12\right)\right.} \\
& \left.-\left((-1)^{l}+1\right)\left(16 l^{4}+73 l^{3}+106 l^{2}+64 l+24\right)\right]\left(\frac{2 q k}{m_{e}^{2}}\right)^{l},
\end{aligned}
$$

where $\Gamma$ is the gamma function. It is clear that the series readily produces, after integration upon the thermalized momenta, the low-energy expansion

$$
\begin{aligned}
f\left(q T \ll m_{e}^{2}\right) & =-\frac{e^{4} T^{2}}{\pi^{4}} \sum_{n=1}^{\infty} \frac{\Gamma^{2}(2 n) \Gamma(2 n+1) \zeta(2 n+2)}{\Gamma(4 n+1)} \frac{4 n^{3}+12 n^{2}+5 n+1}{(n+1)}\left(\frac{4 q T}{m_{e}^{2}}\right)^{2 n} \\
& =-e^{4}\left(\frac{11}{4050} \frac{q^{2} T^{4}}{m_{e}^{4}}+\frac{208 \pi^{2}}{212625} \frac{q^{4} T^{6}}{m_{e}^{8}}+\ldots\right)
\end{aligned}
$$

where $\zeta$ stands for the Riemann zeta-function. This gives the expression Eq. (1.3) for the leading modification of the speed of low-energy photons. Further higher order terms can be obtained without trouble. For arbitrary $q$ the computation is much more involved since we need to sum the above series [10]. This is sketched in the Appendix, the basic idea being to go back to (5.4) and subdivide it into simpler series which can be summed using logs, dilogs and trilogs. The result leads to a high-energy expansion whose first term is

$$
f\left(q T \gg m_{e}^{2}\right)=\frac{1}{48 \pi^{2}} e^{4} T^{2} \ln ^{2} \frac{q T}{m_{e}^{2}}+\mathcal{O}\left(T^{2} \ln \frac{q T}{m_{e}^{2}}\right)
$$

leading to

$$
v\left(q T \gg m_{e}^{2}\right)=1-\frac{\alpha^{2}}{6}\left(\frac{T}{q}\right)^{2} \ln ^{2} \frac{q T}{m_{e}^{2}} \quad, \quad T \leq q .
$$

In the process of obtaining this limit, a non-trivial cancellation of $\ln ^{3}$ takes place.

The summary of the above computation is that low-energy corrections are of the same type as already obtained at one loop order (cf. Eq. (4.9)), but this is not so at high energies. Indeed Eq. (5.7) has an IR-sensitive $\ln ^{2}$ factor which is not present in Eq. (4.10). The electron mass plays the role of an IR regulator and the limit $m_{e} \rightarrow 0$ cannot be taken. This is consistent with the fact that the $m_{e} \rightarrow 0$ limit is incompatible with the approximations used (which led to $\Pi_{i j, T}^{(1)}=0$ ). The massless theory cannot be obtained from our results and has to be worked out separately. We will do so in the next section for a simpler theory. Notice that Eq. (5.6) does not satisfy Eq. (3.13) so that one cannot 
interpret it as a genuine modification of the speed of light but rather to one corresponding to an induced high-energy mass which runs according to

$$
\lambda^{2}\left(q T \gg m_{e}^{2}\right)=\frac{\alpha^{2}}{3} T^{2} \ln ^{2} \frac{q T}{m_{e}^{2}} .
$$

The high energy correction Eq. (5.7) is never quantitatively more relevant than the lowenergy correction, Eq. (1.3), as

$$
\left(\frac{T}{q}\right)^{2} \ll\left(\frac{T}{m_{e}}\right)^{4}, q T \gg m_{e}^{2} .
$$

Massive theories do not offer better perspectives for obtaining relevant modifications of the speed of light at high energies than at low energies. Furthermore only at low energies is the modification genuine, i.e. unrelated to an induced mass.

\section{Two-loop massless $g \phi^{4}$}

The results we have obtained so far stress the relevant role played by masses in thermal corrections. For QED, $m_{e}$ has either compensated for the dimensions carried by the temperature or played the role of an IR cutoff. It is, therefore, natural to ask the question of what would happen in a theory without any extrinsic mass. This turns out to be a subtle problem at two loops, that we will study in the laboratory of massless $\frac{g}{4} \phi^{4}$, which is substantially simpler than massless QED.

Let us first recall the one-loop results, coming from the tadpole diagram

$$
\begin{aligned}
& \Pi^{(1)}=0 \\
& \Pi_{T}^{(1)}=\frac{g T^{2}}{24},
\end{aligned}
$$

where we have used dimensional regularization, which preserves at zero temperature the masslessness of the theory. At two loops, Umezawa's two component formalism has to be used [18]. This means that Eq. (4.1) is generalized to

$$
D_{T}\left(k, k^{\prime}\right)=\delta^{(4)}\left(k-k^{\prime}\right)\left[\frac{i}{k^{2}+i \eta\left({ }^{1}-1\right)}\left(\begin{array}{cc}
1 & 0 \\
0 & -1
\end{array}\right)+\frac{2 \pi \delta\left(k^{2}\right)}{e^{\frac{\left|k_{0}\right|}{T}}-1}\left(\begin{array}{cc}
1 & e^{\frac{\left|k_{0}\right|}{2 T}} \\
e^{\frac{\left|k_{0}\right|}{2 T}} & 1
\end{array}\right)\right]
$$

Fields are accordingly doubled so that the upper component is the physical field and the lower, the auxiliary (and always virtual) field which only couples to itself with a quartic coupling of opposite sign. Therefore, physical fields only connect to auxiliary ones through the off-diagonal thermal propagator. The zero temperature two-loop contributions are

$$
\Pi^{(2, t)}=0
$$


for the double tadpole, and $(n=4+2 \epsilon)$

$$
\Pi^{(2, s)}\left(q_{0}^{2}-q^{2}\right)=\frac{g^{2}}{24\left(16 \pi^{2}\right)^{2}}\left(\frac{1}{\epsilon}-\ln \frac{q_{0}^{2}-q^{2}}{\nu^{2}}+c_{1}\right)\left(q_{0}^{2}-q^{2}\right),
$$

for the setting sun diagram. The constant $c_{1}$ depends on the precise definition of the regularization scale $\nu$. Eq. (6.4) of course vanishes on the mass shell. Since Eq. (6.1) is constant, the two-loop generalization of Eq. (3.2) is

$$
E^{2}=q^{2}+\frac{g_{0} T^{2}}{24}+\Pi_{T}^{(2, t)}+\Pi_{T}^{(2, s)}\left(q^{2}, q^{2}\right)
$$

where we have made explicit the bare character of the coupling constant for the one-loop contribution.

Consider now the different thermal contributions to the two-loop tadpole diagram. With the help of Eq. (6.2), the two-point loop gives a contribution proportional to

$$
\begin{aligned}
& \int \frac{d^{n} k}{(2 \pi)^{n}}\left[\left(\frac{i}{k^{2}+i \eta}+\frac{2 \pi \delta\left(k^{2}\right)}{e^{\frac{\left|k_{0}\right|}{T}}-1}\right)^{2}-\left(\frac{2 \pi \delta\left(k^{2}\right)}{e^{\frac{\left|k_{0}\right|}{T}}-1}\right)^{2} e^{\frac{\left|k_{0}\right|}{T}}\right] \\
= & \int \frac{d^{n} k}{(2 \pi)^{n}}\left[-\left(\frac{1}{k^{2}+i \eta}\right)^{2}+\frac{4 \pi i \delta\left(k^{2}\right)}{e^{\frac{\left|k_{0}\right|}{T}}-1}\left(\frac{1}{k^{2}+i \eta}+i \pi \delta\left(k^{2}\right)\right)\right],
\end{aligned}
$$

so that, recalling

$$
\delta\left(k^{2}\right) P \frac{1}{k^{2}}=0
$$

only the zero temperature contribution is left. Thus the whole thermal contribution comes from the other loop. Note also that the Feynman integral

$$
\int \frac{d^{n} k}{(2 \pi)^{n}} \frac{1}{\left(k^{2}+i \eta\right)^{2}}
$$

vanishes in dimensional regularization due to a cancellation of UV and IR divergences. As temperature does not affect renormalizability we know that Eq. (6.5) is UV-finite. It might, however, be IR-singular on the grounds of being a massless theory which is, furthermore, considered on the mass shell. It is, therefore, important to tell IR from UV divergences. This we do by introducing where needed the one-loop thermal mass as an IR regulator in the two-loop contributions. In particular, Eq. (6.8) becomes

$$
\int \frac{d^{n} k}{(2 \pi)^{n}} \frac{1}{\left(k^{2}-\frac{g T^{2}}{24}+i \eta\right)^{2}}=\frac{i}{(4 \pi)^{2}}\left(-\frac{1}{\epsilon}-\ln \frac{g T^{2}}{\nu^{2}}+c_{2}\right) .
$$

Here $c_{2}$ is a constant which depends on the precise value of the IR regulator, which is actually arbitrary. Putting everything together, one finds

$$
\Pi_{T}^{(2, t)}=\frac{g^{2} T^{2}}{48} \frac{1}{16 \pi^{2}}\left(\frac{1}{\epsilon}+\ln \frac{g T^{2}}{\nu^{2}}-c_{2}\right) .
$$


It is worth mentioning here that both in Eq. (6.1) and Eq. (6.10) the thermal loop integral has been performed in $n=4$ dimensions. It will immediately become clear why there is no need to dimensionally regularize it.

The thermalized setting sun diagram has two real contributions. One corresponding to one thermalized particle. It is UV-divergent and IR-finite:

$$
\Pi_{T}^{(2, s, 1)}\left(q^{2}, q^{2}\right)=\frac{g^{2} T^{2}}{24} \frac{1}{16 \pi^{2}}\left(\frac{1}{\epsilon}+\ln \frac{q T}{\nu^{2}}+c_{3}\right) .
$$

Again, the thermal loop integral has been performed in $n=4$ dimensions. The other contribution, corresponding to two thermalized particles, is both UV and IR-finite. It is proportional to the integral

$$
\begin{aligned}
I(q, T) \equiv & g^{2} T^{2} \int_{0}^{\infty} d x \frac{x}{e^{x}-1} \int_{0}^{\infty} d y \frac{y}{e^{y}-1} \int_{-1}^{1} d \mu \int_{-1}^{1} d \rho \sum_{\eta= \pm 1} \sum_{\eta^{\prime}= \pm 1} \\
& \frac{1}{\frac{q}{T}\left(\eta x+\eta^{\prime} x+\mu x\right)+\eta \eta^{\prime} x y+y \rho \sqrt{\left(\frac{q}{T}\right)^{2}+x^{2}-2 \frac{q}{T} x \mu}}
\end{aligned}
$$

which reduces to

$$
\begin{aligned}
I(q, T)= & \frac{4 g^{2} T^{3}}{q} \int_{0}^{\infty} d x\left[\left(\frac{q}{T}+x\right) \ln \left|\frac{q}{T}+x\right|+\left(\frac{q}{T}-x\right) \ln \left|\frac{q}{T}-x\right|\right] \\
& \left(\ln \left(1-e^{-x}\right)-\frac{x}{2} \frac{1}{e^{x}-1}\right) .
\end{aligned}
$$

The high-energy and low-energy limits of $I(q, T)$ are

$$
I(q \gg T) \simeq-\frac{2 \pi^{4}}{9} g^{2} \frac{T^{4}}{q^{2}}+\mathcal{O}\left(\frac{T^{6}}{q^{4}}\right)
$$

and

$$
I(q \ll T) \simeq 4 g^{2} T^{2}\left[3 \sum_{n=1}^{\infty} \frac{\ln n}{n^{2}}-\left(2-\frac{3 \gamma}{2}\right) \frac{\pi^{2}}{3}\right],
$$

where $\gamma$ is Euler's constant. Putting together Eq. (6.10), (6.11) and

$$
\Pi_{T}^{(2, s, 2)}\left(q^{2}, q^{2}\right)=\frac{1}{16(2 \pi)^{4}} I(q, T),
$$

we get, from Eq. (6.5),

$$
E^{2}=q^{2}+\frac{g_{0} T^{2}}{24}+\frac{g^{2} T^{2}}{48} \frac{1}{16 \pi^{2}}\left(\frac{3}{\epsilon}+\ln \frac{q T^{2}}{\nu^{2}}+2 \ln \frac{q T}{\nu^{2}}+c\right)+\frac{1}{16(2 \pi)^{4}} I(q, T) .
$$


Recalling the coupling constant renormalization

$$
g_{0}=g\left(\mu^{2}\right)\left(1-\frac{3 g}{32 \pi^{2}}\left(\frac{1}{\epsilon}+\ln \frac{\mu^{2}}{\nu^{2}}+\frac{c}{3}\right)\right),
$$

where $\mu$ is the renormalization point, we find

$$
E^{2}=q^{2}+\frac{g\left(\mu^{2}\right) T^{2}}{24}+\frac{g^{2} T^{2}}{768 \pi^{2}}\left(\ln \frac{g T^{2}}{\mu^{2}}+2 \ln \frac{q T}{\mu^{2}}\right)+\frac{1}{256 \pi^{4}} I(q, T) .
$$

Notice that, due to the high-energy and low-energy behavior of $I(q, T)$, Eq. (6.14) and (6.15) respectively, its contribution to Eq. (6.19) can be neglected, as it is subdominant. It is now also clear why in Eq. (6.5), Eq. (6.10) and Eq. (6.11) there was no need to dimensionally regularize the thermal Feynman integral: as eventually all UV divergences cancel in Eq. (6.19), the $0(\epsilon)$ terms, which one can see that they factorize, do not contribute. The $\mu$-dependence in Eq. (6.19) is of course fictitious, so that we can immediately renormalization group improve it by substituting $\left(g T^{4} q^{2}\right)^{1 / 3}$ for $\mu^{2}$, so that finally (neglecting $I(q, T))$,

$$
E^{2}=q^{2}+\frac{g\left(\left(g T^{4} q^{2}\right)^{1 / 3}\right) T^{2}}{24}
$$

Thus, the whole two-loop contribution is contained in the running of the coupling constant which appears at one loop,

$$
g\left(\mu^{2}\right)=\frac{g\left(\Lambda^{2}\right)}{1-\frac{3 g\left(\Lambda^{2}\right)}{32 \pi^{2}} \ln \frac{\mu^{2}}{\Lambda^{2}}},
$$

with $\Lambda$ the intrinsic mass scale of the theory. Eq. (6.20) with Eq. (6.21) is the main result of this section. The whole two-loop temperature effect is to make the one-loop thermally-induced mass run. For very high values of $q$,

$$
\left(g T^{4} q^{2}\right)^{1 / 3} \leq \Lambda^{2} e^{\frac{32 \pi^{2}}{3 g\left(\Lambda^{2}\right)}}
$$

one starts being sensitive to the Landau pole and perturbation theory breaks down. Below that,

$$
\left(g T^{4} q^{2}\right)^{1 / 3} \simeq \Lambda^{2}
$$

the running is too little conspicuous to the noticed: the whole temperature effect is a constant mass. At much lower values,

$$
\left(g T^{4} q^{2}\right)^{1 / 3} \ll \Lambda^{2} e^{-\frac{32 \pi^{2}}{3 g\left(\Lambda^{2}\right)}},
$$

the running coupling vanishes logarithmically and

$$
E(q) \simeq q-\frac{2 \pi^{2} T^{2}}{9 q \ln \frac{\left(g T^{4} q^{2}\right)^{1 / 3}}{\Lambda^{2}}}, \quad T \ll q,
$$


where we have made explicit that still the conditions corresponding to Eq. (3.5) hold, i.e. the kinetic energy still dominates over the mass. From Eq. (3.5), one finds

$$
v=1+\frac{2 \pi^{2} T^{2}}{9 q^{2} \ln \frac{\left(g T^{4} q^{2}\right)^{1 / 3}}{\Lambda^{2}}} \leq 1, \quad T \ll q
$$

which corresponds to the modification of the speed of a massless particle which has acquired a logarithmically vanishing mass. As $q$ becomes even lower, $q \ll T$, an IR divergence appears in Eq. (6.19). It requires an IR regulator, which comes in both recalling that propagators can be regulated with the one loop thermal mass or recalling that due to the induced thermal mass the situation of the pole is shifted. In any case, $q^{2}$ friezes out at $g T^{2}$ and we find a constant low energy mass

$$
\lambda^{2}(q \ll T)=\frac{4 \pi^{2} T^{2}}{9 \ln \frac{g^{2 / 3} T^{2}}{\Lambda^{2}}},
$$

with the corresponding non-relativistic velocity, Eq. (3.7).

It is worth stressing again that Eqs. (6.19) or (6.20) are IR sensitive results, as also happened, somewhat differently, for the two loop QED results. For $g \phi^{4}$ the two-loop temperature effects are basically absorbed by the running of the one-loop thermal mass, which does so according to the zero temperature one-loop running of the coupling constant. There is, thus, no genuine modification of the speed of the massless particle. We surmise that this result is of some generality, at least for theories like massless QED which have the same asymptotic behavior. They might hold, mutatis mutandis, for asymptotically free theories like pure QCD, although more serious IR problems should be expected for them.

\section{Casimir vs. temperature}

We now turn to the study of the propagation of photons in a Casimir vacuum, defined by the presence of two infinite, ideal conducting plates. The modifications of observable quantities are ultimately related to the careful implementation of boundary conditions. In a way, the same is true for thermal effects. There, periodic boundary conditions are imposed on imaginary time. We shall keep this analogy present all along our presentation.

Let us start by comparing Eq. (1.3) ( propagation in a heat bath) with Eq. (1.4) ( propagation perpendicular to the Casimir plates, $\cos \theta=1$ ). Clearly both expressions are identical with the substitution $2 T \rightarrow a^{-1}$, but for a sign. This is not surprising as temperature corresponds in the imaginary time formalism to periodic boundary conditions and Casimir plates to half-periodic ones in one space direction. As said above, one expects a correspondence between the formalisms which makes this identity apparent. This was

already pointed out by Barton [5]. In the same spirit but within a different formalism we 
want to obtain the analog to Eq. (4.2) for the Casimir vacuum. This will also allow us to gain more understanding of what a modified vacuum is.

The photon propagator for the Casimir boundary conditions $E_{1}=E_{2}=B_{3}=0$ on $x_{3}= \pm \frac{a}{2}$ has been given in ref. [20]. It has the form

$$
\bar{D}_{c}^{\mu \nu}\left(k, k^{\prime}\right)=\delta^{(4)}\left(k-k^{\prime}\right) D^{\mu \nu}(k)+\delta^{(3)}\left(\tilde{k}-\tilde{k}^{\prime}\right) D_{a}^{\mu \nu}\left(\tilde{k} ; k_{3}, k_{3}^{\prime}\right)
$$

where $D^{\mu \nu}(k)$ is the standard free propagator and $D_{a}^{\mu \nu}\left(\tilde{k} ; k_{3}, k_{3}^{\prime}\right)$ is the gauge-independent modification due to the boundaries. The actual form for this second piece of the propagator was gotten in ref. [20] in position space

$$
\begin{array}{r}
D_{a}^{\mu \nu}\left(\tilde{x}-\tilde{y} ; x_{3}, y_{3}\right)=\frac{1}{4} \int \frac{d^{3} \tilde{k}}{(2 \pi)^{3}} e^{-i \tilde{k} \cdot(\tilde{x}-\tilde{y})}\left(-\tilde{g}^{\mu \nu}+\frac{\tilde{k}^{\mu} \tilde{k}^{\nu}}{\Gamma^{2}(\tilde{k})}\right) \frac{1}{\Gamma(\tilde{k}) \sin (a \Gamma(\tilde{k}))} \\
\left(e^{-i \Gamma(\tilde{k})\left|x_{3}+y_{3}\right|}-e^{i \Gamma(\tilde{k})\left|a-x_{3}+y_{3}\right|}-e^{i \Gamma(\tilde{k})\left|a+x_{3}-y_{3}\right|}+e^{i \Gamma(\tilde{k})\left|x_{3}+y_{3}\right|}\right),
\end{array}
$$

where $\Gamma(\tilde{k})=\tilde{k}^{2}+i \eta$. Since $\tilde{k} \equiv\left(k_{0}, k_{1}, k_{2}, 0\right)$, it is clear that momentum is not conserved in the 3-direction, as corresponds to the breaking of translational invariance in this direction. On the other hand, no energy conservation violation takes place at finite temperatures, because the boundary conditions are imposed in imaginary, not real, time.

Note that Eq. (7.1) includes all the effects due to reflection at the boundaries. As mentioned for finite temperatures after Eq. (4.3), one includes in the modified vacuum only those effects on the propagation which cannot meaningfully be separated from the standard vacuum propagation, i.e. those contributions which also conserve energy and momentum. For a Casimir vacuum this means studying the propagation far from the plates. This implies to consider a wave packet localized in a region far from the boundaries so that the limit $k a \rightarrow \infty$ should be taken. From the above expression for the propagator, this limit can be taken, obtaining

$$
D_{a}^{\mu \nu}\left(\tilde{k}, k_{3}, k_{3}^{\prime}\right) \sim \delta\left(k^{2}\right)\left[\delta\left(k_{3}+k_{3}^{\prime}\right)-e^{i \Gamma(\tilde{k}) a} \delta\left(k_{3}-k_{3}^{\prime}\right)\right], \quad k a \rightarrow \infty .
$$

In the above limit only two directions are coherently enhanced, one of them forward and which thus conserves momentum. This is the contribution which one cannot separate from $D^{\mu \nu}$ and is, then, included into the Casimir vacuum structure. This leads to the operative propagator

$$
D_{c}^{\mu \nu}\left(k, k^{\prime}\right)=\delta^{(4)}\left(k-k^{\prime}\right)\left[-\frac{i g^{\mu \nu}}{k^{2}+i \epsilon}+2 \pi\left(\tilde{g}^{\mu \nu}-\frac{\tilde{k}^{\mu} \tilde{k}^{\nu}}{\Gamma^{2}(\tilde{k})}\right) \frac{\delta\left(k^{2}\right)}{e^{-2 i \Gamma(\tilde{k}) a}-1}\right]
$$

where $D^{\mu \nu}(k)$ has been written in the Feynman gauge and $\tilde{g}^{\mu \nu}=g^{\mu \nu}+\delta^{\mu 3} \delta^{\nu 3}$.

The similarity of Eq. (7.4) with Eq. (4.1), with the substitution $2 T \rightarrow a^{-1}$, catches the eye, except for the fact that in Eq. (7.4) the exponential has an imaginary exponent 
and that the tensor in the $a$-dependent part of Eq. (7.4) is a projector over physical helicities only in the 3 -direction. This explains the $\cos \theta$ dependence of Eq. (1.4), while the imaginary exponent implies a Wick rotation when the loop integral is performed in the Casimir case which did not happen for the temperature vacuum, leading to an analytic continuation $q^{2} \rightarrow-q^{2}$. Our results Eq. (5.5) and Eq. (5.6) would, then, become

$$
f\left(\frac{q}{a} \ll m_{e}^{2}\right)=\frac{11}{4050} e^{4} \frac{q^{2}}{\left(a m_{e}\right)^{4}}
$$

and

$$
f\left(\frac{q}{a} \gg m_{e}^{2}\right)=\frac{1}{48 \pi^{2}} e^{4} T^{2} \ln ^{2} \frac{q}{a m_{e}^{2}}
$$

leading to

$$
v\left(\frac{q}{a} \ll m_{e}^{2}\right)=1+\frac{11 \pi^{2}}{8100} \alpha^{2}\left(\frac{1}{a m_{e}}\right)^{4}>1
$$

and

$$
v\left(\frac{q}{a} \gg m_{e}^{2}\right)=1-\frac{\alpha^{2}}{6}\left(\frac{1}{a q}\right)^{2} \ln ^{2} \frac{q}{a m_{e}^{2}}<1,
$$

always for propagation perpendicular to the plates.

The low-energy expression, with $v>1$, has been interpreted in section 2 as due to a decrease in the vacuum energy density. The high-energy expression, with $v<1$, has been interpreted in section 5 as due to a high-energy induced mass which, of course, always decreases the speed of light. Both results, Eq. (7.7) and Eq. (7.8) required, as discussed before, $a q \gg 1$ as well as $a m_{e} \gg 1$, the condition equivalent to coldness which, here, means that the electron Compton wave length should be smaller than the distance between plates. These two restrictions not only do not contradict the inequalities that hold for Eq. (7.7) and Eq. (7.8) but, rather, ensure that the corrections are small so that perturbation theory holds.

On the contrary, for a cold heat bath $\left(T \ll m_{e}\right)$, the other condition, $T \ll q$, does not follow. This, again, shows that a boundary condition in imaginary time does not impose kinematical constraints as does a boundary condition in real space. In fact, $q \ll T$ was considered in Eq. (6.21).

Physically, $v>1$ in Eq. (7.7) can be interpreted, in harmony with the analysis of section 2 , in terms of a decrease in the number of modes, recalling that real boundary conditions suppress modes. Instead, the presence of temperature adds real particles which allow for coherent scattering and, thus, gives $v<1$. On the contrary, the result $v<1$ in Eq. (7.8) can be understood by recalling that in the standard vacuum the contributions of the different modes balance in such a way that photons remain massless but that now, by suppressing some modes, this balance is broken and a high energy mass appears. This certainly slows down the propagation of photons.

It is worth mentioning that examples of repulsive Casimir effects are known. However, a detail analysis [21] show that the energy density remains lower and, consequently, the speed of light is faster than $c$. 
For gravity, the result $v>1$ [2] [8] can be interpreted similarly as a decrease in the number of modes, and $v<1$ as the appearance of a mass, following the same line of thought as done above for the Casimir vacuum.

\section{Conclusions}

We have studied, up to two loops, the modifications of the speed of massless particles in a cold heat bath and in other modified vacua, for massive and massless theories, for high and low energies. We have found a unifying expression for the low energy change in the averaged speed of a light valid for all vacua:

$$
v=1-\frac{44}{135} \alpha^{2} \frac{\rho}{m_{e}^{4}}
$$

where $\rho$ is the energy density and where, if the vacuum is a gravitational one, one $\alpha$ has to be substituted by $m_{e}^{2} G_{N}$. It follows automatically that if the vacuum has a lower energy density than the standard vacuum, $\rho<0$ and $v>1$, and viceversa.

An enigmatic consequence of assuming full generality of the equation we have just discussed takes place in cosmology. There, open, closed and critical universes are distinguished by their total energy content. It follows from Eq. (8.1) that the critical universe is singularized as low-energy photons travel in it with speed $c$, due to the cancellation of all vacuum energy contributions.

At one loop the whole temperature effect in the case of a massless theory is just to give a mass. For a massive theory it leads to a genuine change in the speed of the particle at low energies, but suppressed by $\left(\frac{T}{m}\right)^{4}$. It parallels the two-loop results in Eq. (8.1). At high energies, it leads to a change in the speed of the particle as caused by a thermal mass, which only plays a role at high energies. The effect is suppressed by $\left(\frac{T}{q}\right)^{2}$.

At two loops the whole temperature effect in the case of the massless theory chosen, $g \phi^{4}$, is to let the thermally induced one-loop mass run, through the running of the coupling constant. It is an IR-sensitive result which requires an IR cutoff, which we have taken to be the one loop-thermal mass. All the changes in the speed of the massless particle are due to the now running thermal mass. For the massive theory chosen, QED, the twoloop low-energy result reproduces the known modifications, and is suppressed by the same factor as at one loop. The high-energy behavior, however, differs from the one obtained at one loop by a squared logarithmic correction which, furthermore, depends on the mass of the theory in an IR sensitive way. Nevertheless, it can be interpreted, like at one loop, as due to a now running thermal mass.

Finally, the modification of the speed of light due to Casimir plates has been compared and related to the one due to temperature. A field theoretical explanation in terms of modes suggests the following physical picture of why photons move faster between plates than in a normal vacuum, in contrast to what happens in a heated vacuum. Modifications 
of the vacuum that populate it with real or virtual particles introduce coherent scattering which reduces the speed of massless particles. The opposite situation, e.g. Casimir vacuum, corresponds to modifications of the vacuum that eliminate virtual modes and, consequently, their would-be scattering. The speed of massless particles is, then, increased.

The most concise summary of our results states that only low-energy massless particles corresponding to a massive theory show genuine modifications of their speed while remaining massless. All other modifications are mass-related, or running mass-related.

\section{Acknowledgements}

We thank P. E. Haagensen and D. Z. Freedman for his comments on the manuscript. Financial support to this work has been granted by CICYT, AEN-93-0695. Both R. T. and J. I. L. acknowledge hospitality at CERN. 


\section{Appendix}

In section 5 , we needed to calculate

$$
f\left(q, T, m_{e}\right)=-\frac{e^{4}}{8 \pi^{4}} \int_{0}^{\infty} \mathrm{d} k \frac{k}{e^{\frac{k}{T}}-1} R\left(\frac{k q}{m_{e}^{2}}\right)
$$

where

$$
R(y) \equiv \sum_{l=2}^{\infty} \frac{\Gamma^{2}(l)}{\Gamma(2 l+2)} 2^{2 l+1} y^{l}\left((-1)^{l}+1\right)\left(1+\frac{2 l^{2}}{(l+1)^{2}(l+2)}\right)
$$

First, we define

$$
R(y)=S(y)+S(-y)
$$

with $S$ containing all the powers in the series

$$
S(y) \equiv \sum_{l=2}^{\infty} \frac{\Gamma^{2}(l)}{\Gamma(2 l+2)} 2^{2 l+1} y^{l}\left(1+\frac{2 l^{2}}{(l+1)^{2}(l+2)}\right) .
$$

This sum can be splitted into three pieces,

$$
S(y)=\frac{1}{y^{2}}\left(S_{1}(y)+5 S_{2}(y)+2 S_{3}(y)\right) .
$$

The first two are easily carried out in terms of logarithms. The third requires changes of variables to get it ready for resumation in terms of trilogarithms. These partial results are

$$
\begin{aligned}
& S_{1}(y) \equiv \sqrt{\pi} \sum_{n=4}^{\infty} \frac{\Gamma^{2}(n-1)}{\Gamma\left(n-\frac{1}{2}\right)} \frac{y^{n}}{n !} \\
&=-y-y^{2}-\frac{2 y^{3}}{9}-2 i \sqrt{y(1-y)} \ln (i \sqrt{y}+\sqrt{1-y})+(1-2 y) \ln ^{2}(i \sqrt{y}+\sqrt{1-y}) \\
& S_{2}(y) \equiv \sqrt{\pi} \sum_{n=4}^{\infty} \frac{\Gamma(n-1) \Gamma(n-2)}{\Gamma\left(n-\frac{1}{2}\right)} \frac{y^{n}}{n !} \\
&=\frac{y}{2}+\frac{7}{2} y^{2}-\frac{2}{9} y^{3}+(1+2 y) i \sqrt{y(1-y)} \ln (i \sqrt{y}+\sqrt{1-y})+\left(-\frac{1}{2}+2 y\right) \ln ^{2}(i \sqrt{y} \\
&+\sqrt{1-y}), \\
& \quad S_{3}(y) \equiv \\
& \quad \sqrt{\pi} \sum_{n=4}^{\infty} \frac{\Gamma^{2}(n-2)}{\Gamma\left(n-\frac{1}{2}\right)(n-1)} \frac{y^{n}}{n !} \\
& \quad+\frac{y}{4}-\frac{53}{4} y^{2}-\frac{y^{3}}{9}+\frac{i}{2} \sqrt{y(1-y)}(1-18 y) \ln (i \sqrt{y}+\sqrt{1-y}) \\
& \quad+4 y \ln ^{2}(i \sqrt{y}+\sqrt{1-y}) \ln (-2 i \sqrt{1-y} \sqrt{y}+2 y) \\
& \quad-4 y \ln ^{2}(i \sqrt{y}+\sqrt{1-y}) L_{2}\left[(\sqrt{1-y}+i \sqrt{y})^{2}\right] \\
& \quad+2 y L_{3}[(\sqrt{1-y}+i \sqrt{y}]-2 y \zeta(3),
\end{aligned}
$$


where we have used the notation

$$
L_{n}(y)=\sum_{k=1}^{\infty} \frac{y^{k}}{k^{n}}
$$

Collecting this partial results we obtain

$$
\begin{aligned}
S(y) & =\frac{1}{y^{2}}\left\{2 y-10 y^{2}-\frac{14}{9} y^{3}\right. \\
& +(4-8 y) i \sqrt{y(1-y)} \ln (i \sqrt{y}+\sqrt{1-y}) \\
& +\frac{8}{3} y \ln ^{3}(i \sqrt{y}+\sqrt{1-y}) \\
& -8 y \ln ^{2}(i \sqrt{y}+\sqrt{1-y}) \ln (-2 i \sqrt{y(1-y)}+2 y) \\
& -8 y \ln (i \sqrt{y}+\sqrt{1-y}) L_{2}\left[(i \sqrt{y}+\sqrt{1-y})^{2}\right] \\
& \left.+4 y L_{3}\left[(i \sqrt{y}+\sqrt{1-y})^{2}\right]-4 y \zeta(3)\right\}
\end{aligned}
$$

It is then easy to compute the limits used in the text,

$$
\begin{aligned}
R(y) \underset{y \rightarrow \infty}{\sim} & -2 \ln ^{2} y \\
& \sim \frac{88}{135} y^{2}+\frac{416}{3375} y^{4}+\ldots
\end{aligned}
$$




\section{References}

[1] S. L. Adler, Ann. Phys. 67 (1971) 599.

[2] I. J. Drummond and S.J. Hathrell, Phys. Rev. D22 (1980) 343.

[3] S. W. Hawking and G. F. R. Ellis, The Large Scale Structure of the Space-Time, Cambridge Univ. Press, Cambridge 1973.

[4] R. Tarrach, Phys. Lett. B133 (1983) 259.

[5] G. Barton, Phys. Lett. B237 (1990) 559.

[6] K. Scharnhorst, Phys. Lett. B236 (1990) 354.

[7] V. B. Berestetskii, E. M. Lifshitz and L. P. Pitaevskii, Quantum Electrodynamics, $2^{\text {nd }}$ edition, Pergamon Press, Oxford 1982.

[8] R. D. Daniels and G. M. Shore, SWAT-93-9, hep-th 9310114.

[9] S. Ben-Menahem, Phys. Lett. B250 (1990) 133.

[10] G. Barton and K. Scharnhorst, J. Phys. A26 (1993) 2037.

[11] R. D. Birrel and P. C. W. Davies, Quantum Fields in Curved Space, Cambridge Univ. Press, Cambridge 1982.

[12] P. Pascual, J. Taron and R. Tarrach, Phys. Rev. D39 (1989) 2993.

[13] R. Pisarski, Phys. Rev. Lett. 63 (1989) 1129 ;

R. Kobes, G. Kunstatter and A. Rebhan, Phys. Rev. Lett. 64 (1990) 2992.

[14] H. A. Weldon, Phys. Rev. D28 (1983) 2007.

[15] P. S. Gribosky and B. R. Holstein, Zeitsch. Phys. C47 (1990) 205.

[16] P. F. Bedaque and A. Das, Phys. Rev. D45 (1992) 2906.

[17] E. Braaten and R. Pisarski, Nucl. Phys. B337 (1990) 569.

[18] A. J. Niemi and G. W. Semenoff, Ann. Phys. 152 ( 1984) 105; Nucl. Phys. B230 (1984) 181 ;

I. Ojima, Ann. Phys. 137 (1981) 1;

H. Umezawa, H. Matsumoto and M. Tachiki, Thermofield Dynamics and Condensed States, North Holland, Amsterdam 1982.

[19] L. Dolan and R. Jackiw, Phys. Rev. D9 (1974) 3357.

[20] M. Bordag, D. Robaschik and E. Wieczorek, Ann. Phys. 165 (1985) 192.

[21] P. Candelas, Ann. Phys. 143 (1982) 241. 